\title{
Rethinking Interference Mitigation Spectrum Efficiency Model in Femtocell Networks using FFR
}

\author{
Vikas Solanki \\ Department of Computer Science Engineering \& IT, \\ Mangalayatan University, Aligarh
}

\begin{abstract}
In a wireless communication cellular network, call activity can be more intensive in some regions than others. In present scenario of society and city development such as metro-cities and NCR-regions in which, high speed, slow speed as well as pedestrian subscribers are available with high-density may decrease the system performance. Splitting the cell size and reduce the transmission distance is one of the effective technique. But, cell splitting technique does not provide efficient solution in the regions in which high speed, slow speed as well as pedestrian subscribers are available. Deploying the femtocell over cellular networks has recently attracted growing interests in academia, industry, and research places. Deploying femtocells over cellular networks is an attractive solution in current scenario for the improvement of cellular network's services providing better coverage and speed. Femtocell provides attractive indoor coverage with high throughput and promising satisfaction of subscribers. Coexisting femtocells and cellular networks lead to severe interference scenarios. However, inter cell interference decreases the system performance, capacity as well as throughput. Therefore, challenge still remaining is to efficiently allocate spectrum to this technology. This paper focuses on interference mitigation techniques in femtocell/macrocell networks and proposes a fractional frequency reuse (FFR) mechanism that leads to increase overall system performance. In particular, the mechanism aims to maximize throughput via a variety of combinations between inner cell radius and frequency allocation to the cellular networks. Additionally, a position minded frequency allocation to the femtocells targets to further reduce the crosstier interference in femtocell networks.
\end{abstract}

\section{General Terms}

Heterogeneous networks, Interference mitigation, Interference avoidance.

\section{Keywords}

FFR mechanism, Femtocell, Cross-tier interference, Co-tier interference, OFDMA

\section{INTRODUCTION}

Massive deployment of small cells is one of the important key solutions to fundamentally enhance the capacity, coverage and performance of the wireless cellular networks. In current scenario, life style and development of metro city and NCR regions will accommodate fast speed, slow speed as well as pedestrian kind of subscribers at same region in same time. In [1], and [2] Vikas Solanki et al were proposed two-tier cellular networks, in which Virtual Direction Frequency Sharing (VDFS) and Horizontal Direction Frequency Sharing (HDFS) schemes take place to handle more intensive region where high and slow speed subscribers are present. Proposed two-tier model restricted fast speed subscribers to

\author{
M. Qasim Rafiq \\ Department of Computer Science Engineering \\ JP University, Anoopshahr
}

overflow in microcell and if slow speed subscribers overflowed to un-preferred tier then it will not return automatically, until it forced to overflow for serving the new/handoff calls in macrocell. In this system a fast moving calls do not shift to microcells to avoid more handoff. Thus, overall system can avoid more call dropping probability. The scheme Horizontal Direction Frequency Sharing works only in upper-tier with $\mathrm{k}$ neighbouring cells, when VDFS scheme fails and not able to provide the service for arriving calls.

A survey studies in [3] show that more than $50 \%$ of voice calls and more than $70 \%$ of data traffic originate in indoor/ offices/ market places. Another survey in [4] shows that $30 \%$ of business and $45 \%$ of household users experience poor indoor coverage. The new multimedia services and high data rate applications intensifies the need of good quality indoor coverage. Hence, providing good quality indoor voice and data services is of great importance. This would also be beneficial for the cellular operators in the form of increased revenue and reduced churn. Therefore, improving data rate and QoS at indoor, residential/ market places is important in developing next generation wireless networks. However, due to the large attenuation loss from outdoor base station to indoor subscribers, the traditional cellular system can hardly satisfy the demand for high data rates in indoor environments.

Femtocells were introduced in the indoor environment for signal booster and other services for end users. Femtocells are deployed in the existing macrocell network coverage to enhance network coverage in an indoor environment. Fig. 1 shows the typical femtocell architecture. In [5], O.A. Akinlabi et al. discussed about femtocell in an indoor environment to enhance QoS and high performance to the cellular networks, where the path loss was analyzed within the indoor environment. In this paper, researchers present the simulation base on the signal and the path loss model in the indoor environment to show that femtocell enhanced indoor communication with improved signal strength without interference. It is also observed that the subscribers are near to the base station for smooth and quality of services provided by the service provider. To accommodate the rapid growth in wireless data traffic for business/ home places outdoor and indoor environment, femtocell promising the better and attractive coverage, capacity with high throughput. Femtocell is a special type of home enabled Node Base (HeNB) station which is small in size that can be organised by users and connected with cellular backbone networks through wired broadband link/ one or more of the following backhaul networks technology such as DSL, ADSL, broadband internet connection, Metro Ethernet, WiMAX at home/office/market places [6]. Femtocell base station (FBS/ HeBS) also known as femtocell access point (FAP) is fully user installed technology, therefore reduces infrastructure, maintenance, and operational cost of the operator with high coverage, 
throughput and user satisfaction. Femtocells share same bandwidth with existing macrocell/ microcell and/ or accessible licensed bandwidth (e.g. T V system), therefore efficient resource management and allocation schemes are the critical research issue to reuse the spectrum band and mitigate the co-tier/ cross-tier interference in cellular-femtocell networks. In addition, spectrum allocation schemes and FFR mechanisms must be performed in such a way that helps in reducing interference with high throughput and user satisfaction. Access control strategies in femtocells are employed in three ways: one is allowed to connect only closed subscriber group under closed access, while any subscribers can connect to femtocell in the open or hybrid access. The network capacity can be improved by open/ hybrid access strategies because whatever spectrum coverage decreases may overcome by allow the subscribers to be connected nearby femtocell. As in open/ hybrid strategy, the open subscribers can connect to femtocells, therefore it may share the load of overlaid macrocell/ microcell [7]. The negative aspect of open access strategy is that it allows more handoff and exigent in commercial aspect to the operator, because non-subscribers are avail the services as their own femtocells without any return.

There are some existing works that address various interference mitigation mechanisms in femtocell networks. One of the various attractive solutions of mitigate the interference is power management. Adjusting the power transmission level of the FBSs as per requirements of the geographical area, and evaluating their impact on neighbour femtocell and overlaid macrocell/ microcell for an interference perspective may be fairer and efficient interference mitigation mechanism. Such configuration ensure the access of services to achieve attractive performance regardless of position in the networks for both femtocell and macrocell/ microcell subscribers. Second is frequency allocation mechanism. Allocating different fractional frequency to subscribers served by the macrocell base station (MBS/ MeHB) and HeBS protects the subscribers by interference. Currently, orthogonal frequency division multiple accesses (OFDMA) has dealt to interference mitigation and fined large attention for radio transmission technology of the next generation femtocell networks, such as 3rd generation Partnership Project (3GPP) Long Term Evolution (LTE). However in order to get high spectral efficiency, frequency reuse factor (FRF) of OFDMA cellular system is set as one. The subscriber equipment (SE) working on same time-frequency resource band with other SE in neighbour cells, than it will feel a large inter-cell interference (ICI) [8]. It will degrade the system performance, especially near the cell edge site SE. Interference mitigation techniques may be classified as follows: one is interference coordination, second is interference cancellation and third is interference randomization.

Fractional frequency reuse scheme [9] is an interference coordination technique. It restricts the cell resources assignment at the cell boundary and coordinates the restriction of resources among the neighbour cells. And hence due to this scheme, interference becomes low in the adjacent cell boundary results in enhance the system performance. Frequency allocation is an interference cancellation technique. This is preferable, when macrocell/ microcell utilize inter-cell interference cancellation and leaving unexploited frequency for the femtocell utilization. Frequency hopping scheme is an interference randomization technique. It changes the transmit frequency every time slot to obtain the different experienced channel frequency response and different interference, which can get frequency diversity and etiolate the interference of the single SE to improve the link performance. But the frequency hopping scheme is not highly compatible with the frequencyselective scheduling.

For a high performance cellular system, the peak bit rate of cell-edge SE and system throughput are two important criteria, in which the former is more important. No matter where the SE is serving, i.e., regardless in the inner-cell or at the cell-boundary, it has same data rate request. So how to enhance the cell-edge SE's peak rate while keeping the high system throughput is a problem. It is clearly visualized that a FAP would be the typical designed to support hot spot area with cellular access of two to six mobile users in residential or small indoor environments. For commercial environment, it may support with cellular access of six to fourteen mobile users. In [10], assumptions show that about $60 \%$ of voice traffic and $90 \%$ of data traffic will be originated from indoor environments in the near future. Such places may be residential towers/ building, offices, market places, an airport, railway stations and schools/ colleges etc. Therefore, improvement in the indoor coverage area with high throughput is the requirement of next generation cellular networks. However, due to the limited cellular capacity, it might be difficult and too expensive to accommodate this increased traffic demand using the current macro-micro cellular coverage.

The rest of this paper is organized as follows. The advantages of deploying femtocell are described in Section II. The technical challenges in deploying femtocell are discussed in Section III. The interference mitigation strategy is described in Section IV. Section V gives the two proposed interference mitigation schemes using FFR. Section VI contains the conclusion and future work

\section{ADVANTAGES OF DEPLOYING FEMTOCELL}

Among the advantages of deploying wireless technology, femtocells are less-cost self organising plug-and-play, reduced transmission power, backward compatibility with the macromicro cellular networks, portability of devices, and scalable deployment wireless technology. The advantages of the femto-cellular technology can be examined from multiple points of view, such as the subscribers, the manufacturers, the application developers, the network operators, service and content providers. For example, from the user's perspective, customers typically expect high data-rate wireless access at low cost and with good quality of service (QoS). Of course, the key advantage of the femtocells for subscribers is that there is no need for an expensive dual-mode handset; rather, the same single-mode handset is used to access the FAPs and the macro-cellular network. From the wireless operator point of view, the most important advantage of the integrated femto-macro/ micro cellular architecture is the ability to offload a large amount of traffic from the macrocell/ microcell network to the femtocell network. This will not only reduce the investment capital, the maintenance cost, as well as operational costs, but will also improve the reliability of the cellular networks.

Even though there are several technical alternatives to improve the indoor coverage, femtocell appears to be the most attractive approach. Compared with the Fixed Mobile Convergence (FMC) framework, femtocells allow servicing large numbers of indoor subscribers. In contrast with the 3G/ Wi-Fi UMA (Unlicensed Mobile Access) technology, femtocell does not require dual-mode handset. Furthermore, 
another drawback of Wi-Fi is its use of the increasingly crowded unlicensed ISM band that causes significant interference. Finally, repeaters (or signal booster) improve the

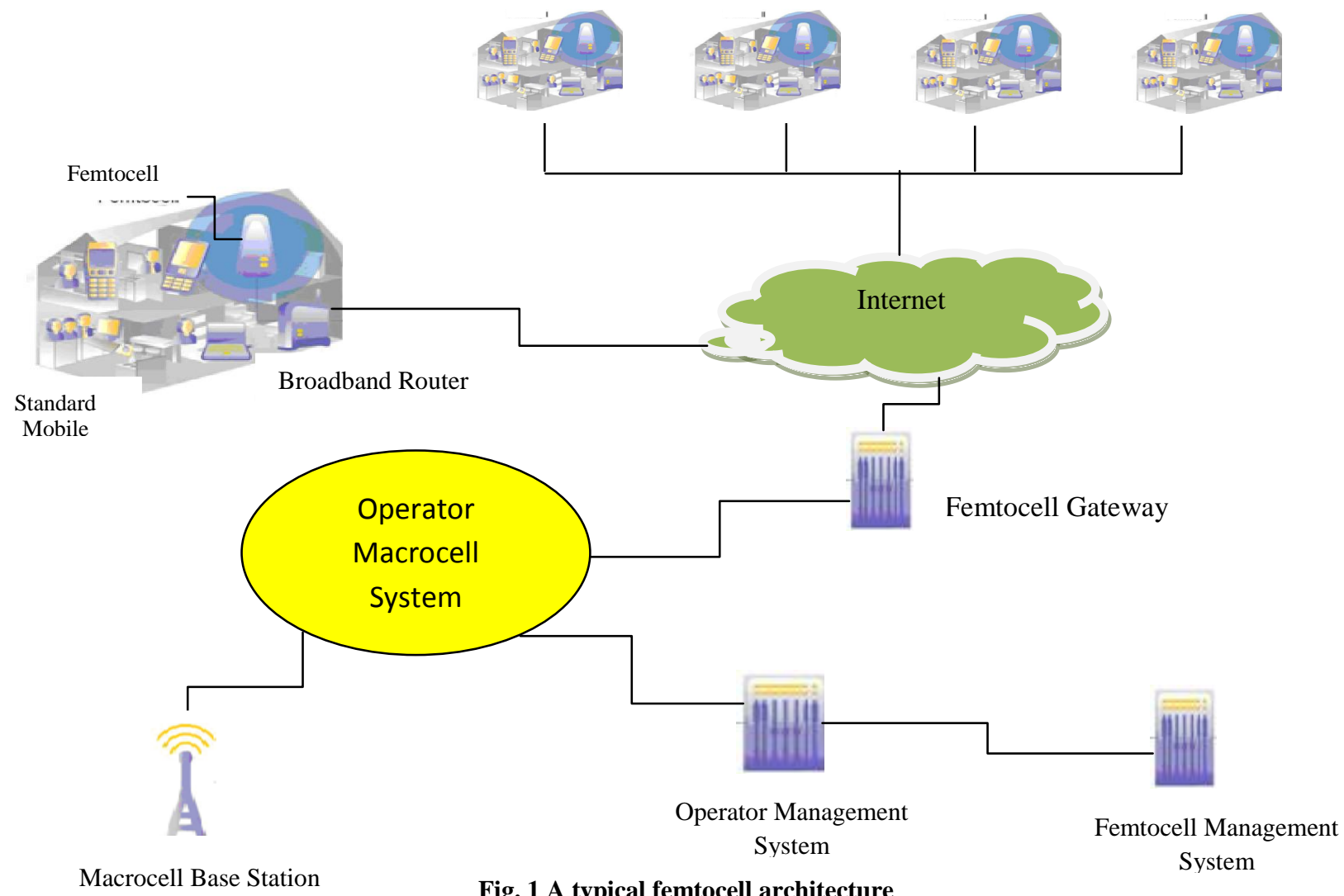

wireless access coverage, but not the wireless capacity. A repeater need new backhaul connections and only solves the poor coverage problem in remote areas, where fixed broadband penetration is low.

From the point of a network operator and users, the following are the key advantages of the integrated femtocell/macrocell network [3], [5]:-

\subsection{Improved Coverage}

Providing extensive coverage at inside the building or outdoor- public palaces, where cellular networks bandwidth is limited or unavailable has long been a challenge for mobile operators. This is even a more difficult problem for communication at higher frequencies, where radio propagation loss is larger. Femtocells can provide better signal reception within the indoor environment, as FAPs are also located inside the building. Thus, using of the basic concept of spectrum re-use, femtocells can improve the network coverage and increase the network capacity. Especially, femtocells extend the service coverage into remote or indoor areas, where access to a macro/micro cellular network is unavailable or is limited. Of course, improved coverage and access capacity enhances customer satisfaction, and hence allowing the network operator to retain and increase its customer pool.

\subsection{Improved Reliability of Cellular Networks}

Traffic originating indoors can be accommodated into the femtocell networks over the IP backbone; the Cellular Base Station can redirect its resources towards providing services to other subscribers, who have better reception.

\subsection{Reduced Infrastructure and Installation Costs}

Since HeNBs are devices required to be simple, plug-and-play self organising without complex data processing and operation ability home base station and the cost of hardware is much lower than that of macrocell base stations. Femtocells use the existing home broadband connectivity for backhauling the femtocells' traffic. Thus, by steering users' traffic into their own FAPs and away from the macrocells, femtocells reduce the expensive backhaul costs of cellular networks.

\subsection{Sharing Load}

As we know that femtocell are installed with existing macrocell/ microcell and/ or accessible licensed bandwidth that helps in offload the large amount of traffic from the macrocell/ microcell network to the femtocell network. Sharing the load helps in increasing the traffic as well as reliability of the cellular networks. 


\subsection{Improved Performance and User Satisfaction}

Deployment of femtocell at indoor-residential/ public places results in improving coverage, performance and user satisfaction. Femtocell helps in offload the macro-micro cellular networks and improve the QoS result in attracts the subscribers that may increase the number of connection for the operators.

\subsection{Security}

The entire smart home appliances are linked to the FAP, for the purpose of communication and security of the home or office.

\subsection{Less Transmission Power}

Transmission power is significantly smaller, as compared to the transmission power of the cellular network that is required to penetrate into buildings. Smaller transmission power results in decreased battery drainage of the mobile devices, prolonging the devices' lifetime. Furthermore, decrease in the transmission power reduces inter-cell interference, increasing the signal-to-interference-ratio. This, in turn, improves the reception, increasing capacity and coverage.

\subsection{Power Saving}

Since femtocells target indoor coverage, therefore electricity bill is no more the responsibility of the operator's.

\subsection{Health and Safety}

Manufacturer of Femtocell has indicated that no health threat had been reported during the use of Femtocell in the indoor environment. Presently the principle of radio frequency radiation is set to ranking, which allow radio transmission of several forms to be used, but at a level where current scientific research shows that there are no unreasonable risk in regard to the usage of Femtocell at the indoor environment. Femtocell fulfill all the safety limits that are applied to other wireless device such as mobile phones, Wi-Fi access points, Bluetooth devices, etc. Thus, these safety limits have been recognized by the International Commission on Non-Ionizing Radiation Protection (ICNIRP) and also authorized by the World Health Organization (WHO) and widely approved by governments around the world.

\subsection{Provisioning of QoS}

The radio path loss close to the cell-edge of a macrocell/ microcell can be quite severe. Femtocells can in particular improve the QoS for subscribers with poor macrocell/ microcell reception.

\section{TECHNICAL CHALLENGES IN DEPLOYING FEMTOCELL}

This section addresses the key technical challenges would be encountered in deploying femtocell management. As deploying femtocell arises so many advantages to both subscribers and operators, still these have many challenges and issues:

There are so many issues for keeping femtocell costs as low as possible for competing against the ubiquitous Wi-Fi technology. The main challenge for the femtocell deployments is the interference with the macrocell/ microcell base station. Other femtocell functions are addressed, including resource management, spectrum allocation management, providing QoS over an internet backhaul and allowing access to femtocells [3]. Handover and mobility also very important aspect in femtocell networks, as there are different types in femtocell handover from/ to macrocell. Furthermore, power consumption is very important consideration to be taken into account in the next generation wireless networks. In metro city and NCR regions, where high speed and slow speed subscribers are compete for call activity as well as high data rates, may be serviced by two-tier networks as discussed in [1], [2]. The deployment of femtocell networks in such places brings new changes in cellular networks. The new architecture composes of three layer network or three-tier networks namely macrocell layer, microcell layer, and femtocell layer. The first two layers (twotiers) are the traditional cellular architecture and third layer composes of different smaller cell that can be planned to be deployed for efficient coverage, high data rates and better performance of the system. This architecture networks bring in some challenges to the networks system as well as operators. As discussed earlier that femtocell uses same spectrum band of existing cellular networks, therefore, the various transmitters within the same frequency band might not be able to differentiate which one of the transmitter it listening to. These arise to interference in mobile communication cellular systems. Therefore interference mitigation schemes play vital role to improve the performance of the system. Hence, in absence of interference mitigation schemes in twotier femtocell or three-tier femtocell networks, dead zone may be created in the existing cellular networks, causing disturbance in the service of femtocell. Due to this problem, a cellular-femtocell networks performance and user satisfaction may goes down. Therefore, interference management is the main issue in the technical challenges for overcomes the problem causing copes up of limited bandwidth to accommodate more subscribers with better performance and user satisfaction. The others challenges faced by femtocell apart from interference are self-organization, access methods, synchronization and timing, location tracking, security, mobility movement and handover. But the main concern is interference management which is the important issue in the networking operation.

\subsection{Frequency and Interference Management}

For better QoS, performance and higher capacity in integrated two-tier or three-tier networks, frequency allocation and interference mitigation schemes are needed. Intelligent and automated radio-frequency planning is needed to minimize interference for random and unknown installation of femtocells. In 3GPP [11], different kind of deployment configurations have been taken for FAP. Dedicating a channel is possible for femtocell subscribers operating in not-in-used spectrum band of the macrocells/ microcells. Since in this approach, there is no interference between femtocell subscribers and macrocell/ microcell subscribers. It is an optimal solution that avoids the interference problem altogether. But this solution also reduces the spectral efficiency, so it is quite expensive. Hence, sharing frequency bands between femtocells and Macrocell seems to be an appropriate solution, but it is technically more challenging.

The main types of interference in two and/or three tier architecture network, both in uplink and downlink are crosstier and co-tier interference. Fig. 2 illustrates the both cross tier and co tier interference in deployment of femtocell.

\subsubsection{Cross-tier interference}

Cross-tier interference may occur in different networks layer. This type of interference is due to deployment of co-channel in CDMA at different (overlapping) layer, e.g. the unwanted 
signals by FAP cause interference to downlink of macrocell users and likewise the unwanted signal by macrocell user at the uplink cause interference to FAP user. due to signal received from other unwanted neighbor femtocell. Because deploying femtocell is opportunistic plugand - play self organized technology, therefore it may create

\subsubsection{Co-tier interference}

Co-tier interference is also known as co-layer interference may occur in same network layer. This type of interference is

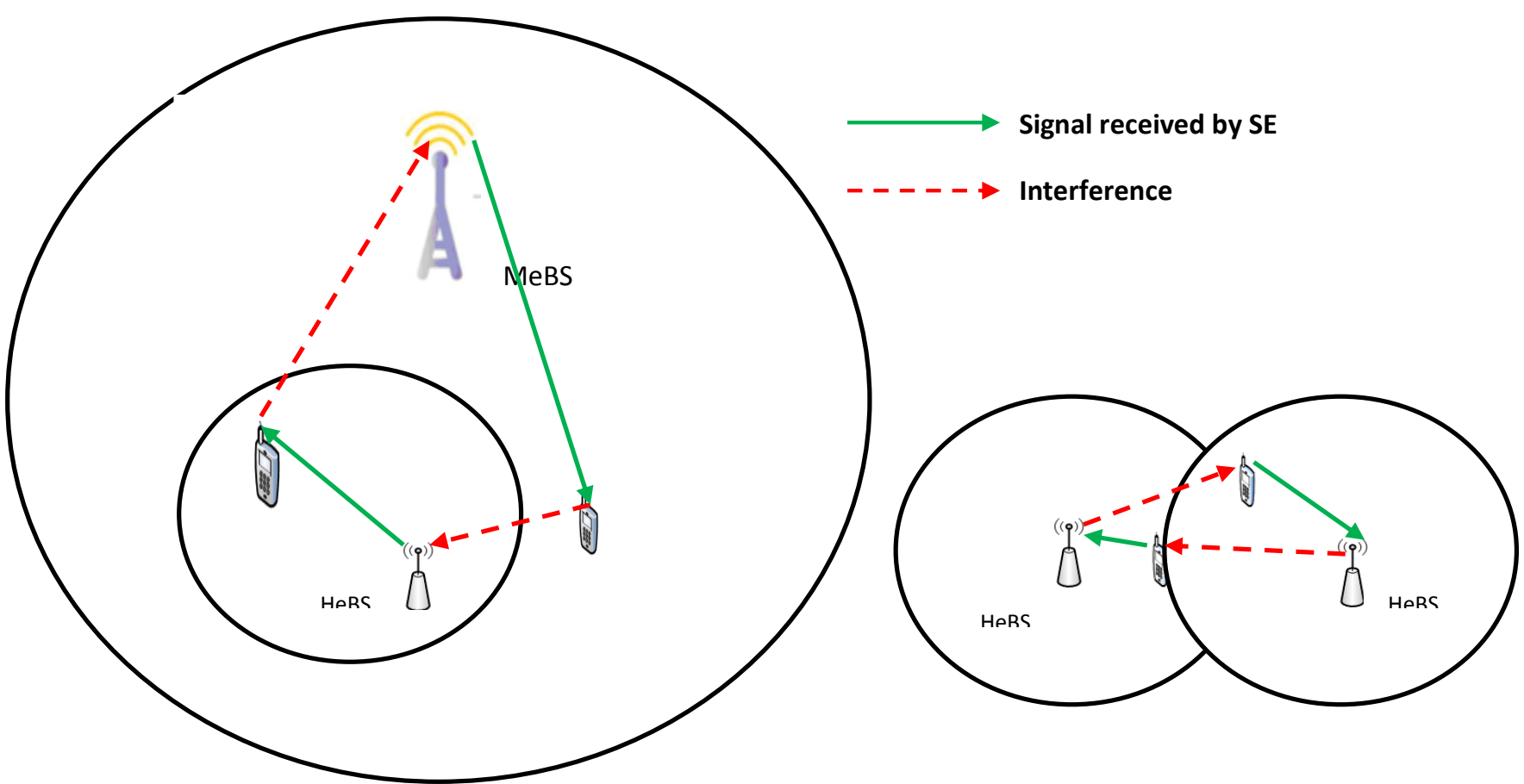

(a) Cross tier interference

(b) Co tier interference

Fig. 2 Interference in deployment of femtocell

co-tier interference that reduces the system performance and communication quality.

\subsection{Self Organisation}

Basically FAP is a plug-and-play, self organising device, and therefore may be turn on and turn off at any time. The main motive beside the plug-and-play device is to reduce the operational expenditure by removing any human involvement in operational task of networks. Self organisation involved self-configuration, self-optimization, and self healing. Hence it is a device that may work on automatic mode to support less human involvement to improve QoS.

\subsection{Access Methods}

Femtocell base stations have a list of its subscribers to prevent the usage, and limit the access of femtocells against illegal users. Three types of access control methods in which femtocells can be configured to allow or block the users are thoroughly proposed [3], [7]:

\subsubsection{Closed Access Femtocell}

A closed access femtocell has a list of subscribed home users for privacy and security, who have licensed to use the femtocell.

\subsubsection{Open Access Femtocell}

On the other hand, open access femtocells provide service to macrocell/ microcell users, if they are pass within the range in.Radio interference is managed by allowing strong macrocell/ microcell interferers to communicate with nearby femtocells. Although open access reduces the macrocell/ microcell load, the higher numbers of subscribers communicating with each femtocell will strain the backhaul to provide sufficient capacity and raise privacy concerns for home users.

\subsubsection{Hybrid Access Femtocell}

Hybrid access femtocell allows to both subscribers home subscribers as well as roamers. Since femtocells are typically marketed as offering flat-rate calling, open access will need to differentiate between the zero-tariff home users from the payper-minute visitor. For both reasons, operators are looking at hybrid models where some of the femto's resources are reserved for registered family members, while others are open for roamers. However, femtocell subscribers are always get priority over cellular (microcell/ macrocell) networks subscribers to access the spectrum under hybrid access.

\subsection{Timing and synchronization}

Timing and synchronization is one of the major challenges for femtocells because synchronization over IP backhaul is difficult, and inconsistent delays may occur due to varying traffic congestion. 


\subsection{Location Tracking}

Government mandated Emergency-911 services require operators to provision femtocells for transmitting location information during emergency calls. Femtocell location may be obtained by either using GPS inside femtocells (added cost with possibly poor indoor coverage), or querying the service provider for location over the backhaul, or gathering information from the cellular networks providing the femtocell falls within the cellular-cell (macrocell/ microcell) radio range, or even inferring the location from the mobile position (estimated by the macro/ micro network) at handoff to the femtocell. Ethical/ legal dilemmas can arise on whether a femtocell should service macrocell users with poor outdoor coverage for making emergency calls, if they are located within its radio range. In open access networks, this problem can be solved by handoff. Closed access femtocells should be provisioned to allow communication with unsubscribed users in the event of emergencies [3].

\subsection{Security}

Security in femtocell networks is one of the challenging key issues. In the case of open / hybrid access mode, security issue is more challenging as the subscriber's private information needs to be protected. The femtocell network is highly sensitive and risky. For example, the private information of subscriber move over the backhaul internet connection may be hacked, which would be break confidentiality and blab privacy [12]

The femtocells are also prone to Denial of Service (DoS) attacks. A hacker can overload the link between a FAP and mobile core network. This would be prohibited the subscribers to make the connection with the core network and result in femtocell service would be failed. In case of close access mode, security is also necessary to prevent unwanted subscribers by utilizing the resources and services of femtocell networks. There are number of threats also pointed in [13] such as eavesdropping, main-in-middle attack, and compromising subscriber's access list. Deploying the femtocell in more dense area would be needed more and more security.

Due to these threats, operators are required to give the Internet Protocol Security (IPSec) to the link between FAP and operators core network. To provide the security in core networks, a secure tunnel is established between the FAP and the security gateway. All the data to and from the femtocell move through the secure tunnel, and hence travelling the data becomes more secure [14].

\subsection{Mobility Management}

In conventional cellular networks, handoffs are initiated when subscribers go in the coverage area of neighbour cells and frequency power of current attachment base station goes down the threshold value. However, given the coverage size of open access femtocells, this occurs more often than in the macrocell case, hence increasing network signaling. Different handoff management schemes are required to allow non-subscribers for staying longer periods in nearby femtocells. Furthermore, a Hierarchical Cell Structure (HCS) can also be used in differentiate between macrocell and femtocells. In this way, the signaling across layers can be minimized and the neighbouring cell list the users scan when performing the handoff [15].

In principle, femtocells treat as other base stations and, therefore may be utilized the present mobility procedures. But, femtocell mobility results in number of unique challenges that require special attention. Standards bodies such as 3GPP have devoted considerable attention towards femtocell mobility issues [16]. Some methods are also being developed for vertical handovers between femtocells and noncellular access technologies such as Wi-Fi [17], [18]. Conceivably the most difficult aspect of femtocell mobility is that femtocells are not directly connected into the core network where mobility procedures are usually coordinated. Due to delay connection in the core network result in significant handover signaling delays. Moreover, for similar architectural reasons, CDMA femtocells suffer from a further limitation that they are typically unable to share a Radio Network Controller (RNC) with a macrocell or other femtocell for coordinating soft handovers. Several works have begun considering architectural changes in the core network and femtocell gateway functions to address these mobility issues [19], [20], although the subject remains an active area of research. Femtocells and picocells also result in much more dense deployments, which complicates base station discovery; a key initial step in any handover.

Another complicating factor in femtocell mobility is the support for a new standard called Selected IP Traffic Offload (SIPTO). SIPTO allows internet traffic to flow from the femtocell directly to the internet, bypassing the operator's core network [21]. In typical cellular networks, data is routed through a fixed gateway that provides a mobility anchor and constant IP point of attachment to the public Internet. Whereas SIPTO is used to selectively offload the IP Traffic over internet by avoiding the mobile core network for the Data traffic. Therefore, with SIPTO, IP traffic may be routed directly to the femtocell, offloading traffic from the operator's core network. In such cases, however, each connection to a femtocell results in a different network point of attachment, possibly with a different IP addresses. Then, mobility must be handled at other place [22].

\subsection{Handover}

Femtocell handover techniques need to ensure that seamless coverage is felt by the subscribers, when moving onto or off a femtocell. Femtocell handover is more challenging than normal conventional cellular handover because the backhaul network is different and there is also little possibility of direct communication between the femtocell and the macrocell/ microcell. The ability to seamlessly handover between the femto and the conventional cellular networks is a key element of the integrated architecture. Make-before-break handover may be necessary for the femtocell environments, because handovers will occur frequently due to the small size of the femtocells. There are four scenarios for handover in cellular femtocell integrated networks:

\subsubsection{Inbound}

This is the handoff typically will occur from the macro-cell or standard cellular network to the femtocell.

\subsubsection{Outbound}

This is the handoff typically will occur from the femtocell to the macro-cell or standard cellular network.

\subsubsection{Femtocell to Femtocell}

There will be situations where handover will occur between one femtocell and another neighbour femtocell. This will be commonplace in offices/ schools/ airports that may have a number of femtocells to give continuous coverage within a building. 


\subsubsection{Macrocell to Macrocell}

This is the handoff typically will occur by the fast speed subscribers or the subscribers switch their connection in other neighbour cell serving by the macrocell.

\subsection{Cell Association and Biasing}

Assign the subscribers to appropriate base station is a key challenge in the heterogeneous networks. A method in [23] does in fact maximize the Signal-to-Interference-Plus-Noise Ratio (SINR) of each subscriber is to simply assign the each subscriber to the strongest base station signal it receive. However, simulation results and field trials have shown that such an approach does not increase the overall throughput as much as hoped, because many of the small cells will typically have few active users.

This motivates biasing, whereby subscribers are actively pushed into the small cells. It has potential for triumph because mobile connected to small cell for larger fraction of time and frequency slots, despite of large cell does maximum SINR hit for that mobile. Furthermore, the macrocell and microcell reclaim against time and frequency slots which would have occupied by the subscriber actively pushed into the small cell. Biasing is particularly attractive in OFDMA networks since the biased user can be assigned orthogonal resources to the macrocell, so the interference is tolerable. But biasing has introduced challenge that includes the use of overhead channels, which are typically common to all BSs in time and frequency and therefore biased subscriber would not be able to listen its channel assignment. The problem can be solved by introducing time-slotting for the control channels [24] or interference cancellation [25].

From a research point of view, a multi-tier architecture including femtocells gives attractive option to revisit cell association and load balancing rules framed only for macrocell cellular networks. In particular, it is not clear that how much biasing is "optimal": it clearly depends on (i) the performance/ QoS metric of interest, (ii) how users and the various base stations are distributed in geographical area, (iii) traffic patterns in space-time, and (iv) the amount of adaptivity and side information the mobiles and small cell base stations are able to exploit.

\section{INTERFERENCE MITIGATION STRATEGY}

The various types of interference will be introduced in the cellular networks, when new femtocells are deployed with conventional cellular networks. Such architecture consists of two layers/ two tiers. The first layer is the conventional macrocell/ microcell networks tier and on the other hand second layer is the femtocell networks tier. Femtocell networks architecture brings forth new problems and design challenges with advantages to be deployed with conventional cellular networks as discussed earlier in section III and section II respectively. Among these challenges, interference mitigation in macro-femto cellular two tiers networks is of importance. Femtocell is preferred to be deployed in co channel fashion and therefore using same spectrum band as the macrocell/ microcell to accommodate large number of subscribers in allotted spectrum band. This in result gives challenges to mitigate co-tier and cross-tier interference in the networks. Interference in the network returns dead zone, which is prone to reduce the capacity as well as performance and QoS of the networks. The interference problems faced in both uplink and downlink is occurred in deployment of femtocells, which mainly deployed with the CDMA and OFDMA technologies. OFDMA technology provides better mechanism to avoiding interference in comparison to CDMA due to division of spectrum into small sub channels [26]. Each of these technologies uses different techniques to manage the co-tier and cross-tier interference. The research is still going on in this area and many schemes have been proposed available in the literature. Some of the main schemes are available in literature as follows:

A femto-aware spectrum arrangement scheme to avoid uplink cross-tier interference between a macrocell and femtocells proposed by $\mathrm{Yi} \mathrm{Wu}$ et al., Clustering of femtocell, Power control approach, Fractional frequency reuse, Frequency reuse and resource allocation, Resource partitioning, hybrid spectrum sharing, Dynamic spectrum reuse, Split spectrum reuse, Adaptive sub-band allocation, Resource allocation based on traffic, Cognitive radio based resource allocation some popular interference management schemes are summarised and detail analysis is given to highlight their pros and cons in [27]. 3GPP has introduced Long Term Evolution (LTE) femtocell to handle the traffic for indoor users and helps in offload the traffic of conventional cellular networks results in improve the performance of cellular networks. LTE is developed by the 3GPP in order to make sure the effectiveness of its standards in the long term. Recently it is also known as 4th generation technology. LTE is the evolution of 3rd generation mobile technology, also called as Universal Mobile Telecommunications System (UMTS). The main challenges for LTE is to come up with new radio access technology so that high data rates, low latency can be offered.

\subsection{Related Work}

In [28] Talha Zahir et al., summarises the main concepts of femtocells that are covered in literature and the major challenges faced in its large scale deployment. The main challenge of interference management is discussed in detail with its types in femtocells and the solutions proposed over the years to manage interference have been summarised. In addition an overview of the current femtocell standardisation and the future research direction of femtocells have also been provided. It provides an insight into the potential research areas of femtocells that can be explored. In [29] Poongup Lee et al, propose an interference management scheme in the LTE femtocell systems using Fractional Frequency Reuse (FFR). Under the macrocell allocating frequency band by the FFR, the femtocell chooses sub-bands which are not used in the macrocell sub-area to avoid interference. In the proposed scheme, the macrocell is divided into center zone (size of the center zone is 0.63 of corresponding macro coverage) and edge region including three sectors per each region. The reuse factor of one is applied in the center zone, while the edge region adopts the reuse factor of three. The entire frequency band is divided into two parts and one of them is assigned to the center zone. The rest of the band is equally divided into parts and assigned in the three edge regions. Simulation results show that proposed scheme enhances total as well as edge throughputs and reduces the outage probability in overall network, especially for the cell edge users. Interference mitigation between different layers is one of the key issues that need to be resolved for successful deployment of heterogeneous network (HetNets). N. Saquib et al. in [30], Focuses on evaluating three state-of-the-art FFR deployment schemes: strict FFR, soft FFR, and FFR-3 schemes for OFDMA-based two-tier HetNets comprising macrocells overlaid with femtocells. Also, a variation of the FFR-3 scheme, which is referred to as the optimal static FFR 
(OSFFR) scheme, is proposed. A broad comparison among all these FFR schemes is performed by using Monte Carlo simulations considering performance metrics such as outage probability, average network sum rate, and spectral efficiency. Simulation results show that, the average gains in spectral efficiency $(\mathrm{b} / \mathrm{s} / \mathrm{Hz})$ of the network are significantly higher for the proposed scheme when compared to the strict FFR, soft FFR, and FFR-3 schemes. In [31] Tariq, F. et al. presents a new hybrid resource management algorithm (HRMA) for down-links in orthogonal frequency division multiple accessbased systems, with the model analysed for a range of macrofemto deployment scenarios. HRMA employs a dynamic fractional frequency reuse scheme for macro-cell deployment with frequency reuse defined for femto users depending on their location by making certain frequencies locally available based on macro-femto tier information sharing and efficient localised spectrum utilisation. Quantitative performance results confirm the efficacy of the HRMA strategy for various key system metrics including interference minimisation, outage probability and throughput. In [32] Bouras, C. et al. study the interference mitigation techniques in femtocell/macrocell networks and proposed a frequency reuse mechanism that leads to increase overall system performance. In particular, the mechanism aims to maximize throughput via a variety of combinations between inner cell radius and frequency allocation to the macrocell. Additionally, a position minded frequency allocation to the femtocells targets to further optimize the total throughput of the cell. In [33] Sathya, R.V et al. propose a new spectrum allocation method which dynamically allocates the spectrum between Macro and Femto cells and dynamically inside Femto regions based on user traffic demands. This method is applicable only for enterprise cellular deployments in which, all Femtos are deployed in a planned manner. Sathya, V. et al in [34] have considered obstacles (walls, floors) and interference between Macro and Femto BSs. To be fair to both indoor and outdoor users, they designed an efficient placement and power control SON (Self organizing Network) algorithm which optimally places Femtos and dynamically adjusts the transmission power of Femtos based on the occupancy of Macro users in the a high interference zone (HIZone). To do this, they solve two Mixed Integer Programming (MIP) methods namely: Minimize number of Femtos (MinNF) method which guarantees threshold SINR $\left(\mathrm{SINR}_{\mathrm{Th}}\right)-2 \mathrm{~dB}$ for all indoor users and optimal Femto power (OptFP) allocation method which guarantees $\operatorname{SINR}_{\mathrm{Th}}(-4 \mathrm{~dB})$ for indoor users with the Macro users SINR degradation as lesser than $2 \mathrm{~dB}$. M Narsing Yadav et al in [35] presents a novel fractional frequency reuse scheme combined with interference suppression for orthogonal frequency division multiple access network which are used in LTE-A and WiMAX IEEE $802.16 \mathrm{M}$ standardization process. FFR technique partitions each cell into two regions. Allocate different frequency bands each Inner and Outer region. Since the user set inner region are less exposed to inter cellular interference. The frequency resource in each inner region can be universally used. Based on this frequency band allocation. FFR may reduce channel interference and offer large system capacity. The entire mechanism is simulated through existing scenario.

\section{PROPOSED INTERFERENCE MITIGATION SCHEMES USING FFR}

The proposed models of interference mitigation strategy are using FFR mechanism. This method divides the entire frequency band into several bands. Afterwards, each sub-band is assigned to separate macrocell/microcell or macrocell/microcell sub-area. Since the spectrum sub-band assigned separately to macrocell and microcell Mobile Base Station, therefore cross-tier interference can be mitigated in two tier conventional networks. Since femtocells are preferred to be deployed in co-channel fashion and therefore using the same spectrum band as macrocell and microcell two-tier homogeneous cellular communication networks architecture. This paper proposes two interference mitigation strategies based on FFR mechanism: one interference mitigation mechanism using FFR in two tier architecture and second interference mitigation mechanism based on FFR in three-tier architecture.

\subsection{Interference mitigation mechanism using FFR in two tier architecture}

In this scheme, the macrocell is divided into three regions; Inner region, middle region, and outer region. The size of the inner region is 0.34 of the corresponding macrocell and size of rest regions middle and outer are equal 0.33 of the corresponding macrocell. The inner and outer region of macrocell is further divided into three equal sectors as shown in Fig. 3(a). As show in Fig. 3(b), the entire band (B), is thus divided into four equal parts known as B1, B2, B3, B4 satisfying $\mathrm{B} 1+\mathrm{B} 2+\mathrm{B} 3+\mathrm{B} 4=\mathrm{B}$. Three sub bands $\mathrm{B} 2, \mathrm{~B} 3, \mathrm{~B} 4$ are assigned to sectors $\mathrm{S} 1, \mathrm{~S} 2$, and $\mathrm{S} 3$ of inner region respectively by directional (sectored) antenna. The middle region is installed with omnidirectionl-antenna that will cover $33 \%$ area at middle region of macrocell. The sub band B1 is assigned to middle region. The outer region that cover rest $33 \%$ area near to edge of the macrocell have three equal sectors, and thus like inner region, the three sub bands B4, B2, B3 are assigned to sectors S1, S2, S3 of outer region of macrocell respectively. In this scheme femtocell select those spectrum sub band, which are not assigned to current sector/region of corresponding macrocell as shown in Fig. 3. Therefore femtocell applies dissimilar sub band to mitigate the interference from macrocell. The sub band is used in the coverage of macrocell sector as much as possible. Because femtocell have less transmission power in comparison to conventional cellular communication networks, therefore interference among the different layers of heterogeneous networks is considerably prevented. In order to improve the throughput and performance of the subscribers, which are in the outer region near to edge, proposed scheme allocated more number of sub carrier to the femtocells. This scheme has three sub bands to allocate the femto user, which are in outer region, two sub bands to allocate the femto users, which are in middle region and one sub band to allocate femto users, which are in inner region. This scheme has three sub bands to allocate the femto users, which are in outer region that's why the performance of the system may be better. This same technique is applied to overall networks; therefore, the performance of the entire networks is good.

\subsection{Interference mitigation mechanism using FFR in three tier architecture}

In this scheme, first two tiers are conventional cellular networks that taking the advantage of overlapping property for handling the traffic of high speed subscribers and slow speed subscribers. The preferred tier for high speed subscribers is macrocell tier and for slow subscribers is microcell tier. Microcell are deployed and divided into three parts of equal size each. To make the partitioning of microcell regions, the microcell base station $(\mu \mathrm{BS})$ should use three sectorized antennas, each with sector width $2 \pi / 3$. Femtocells are deployed as shown in Fig. 4(a) to offload the microcell and macrocell as well as to improve the coverage and capacity 
in indoor environment.

The entire band (B) which is allocated to two-tier conventional networks is divided into two parts. First part is one.-third (D1) of total band and second part is two-third (D2) of total band (B) as shown in Fig. 4(b). The first sub band (D1) is assigned to microcell networks and second sub band (D2) is assigned to macrocell networks. The microcell is divided into three equal sectors as shown in Fig. 4, therefore the frequency sub band (D1) is further divided into three sub partsB1, B2, and B3and assigned to sector S1, S2, S3 respectively. The macrocell - tier of two - tier architecture is

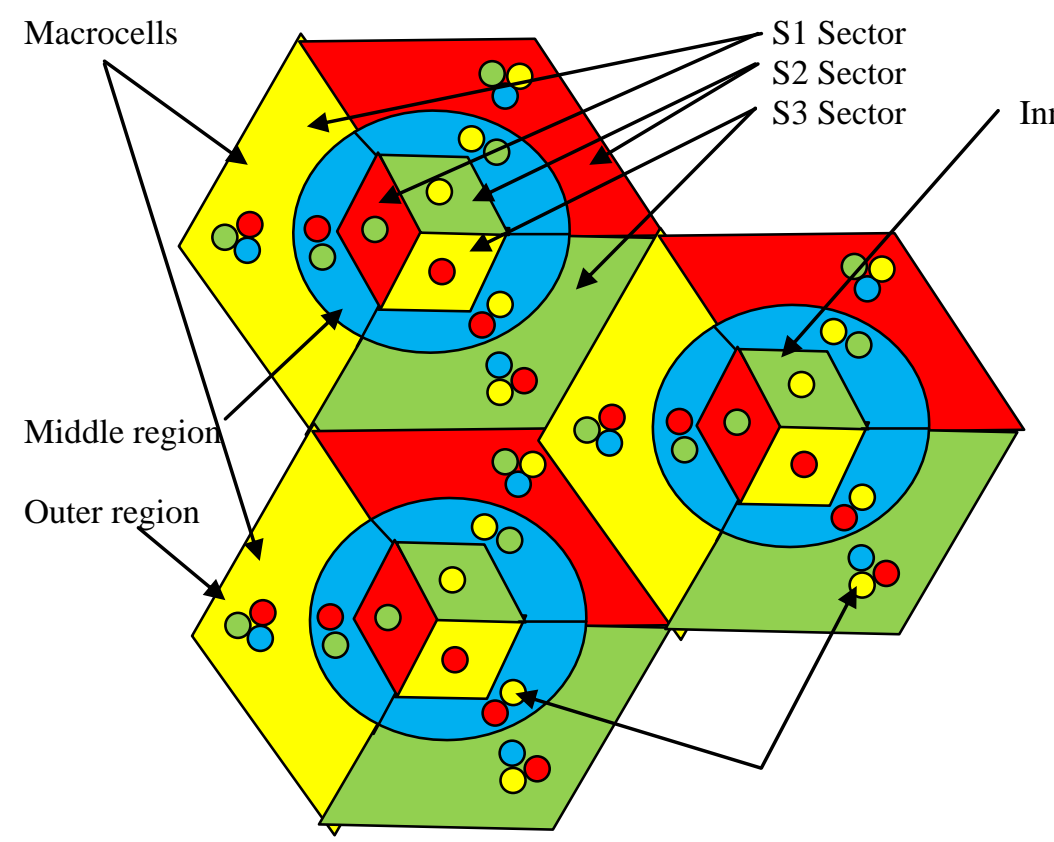

(a) Two-tier femtocell architecture

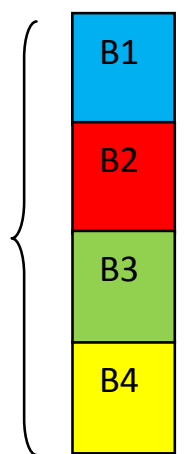

(b) Frequency division

Fig. 3 Interference mitigation scheme using FFR in two-tier architecture

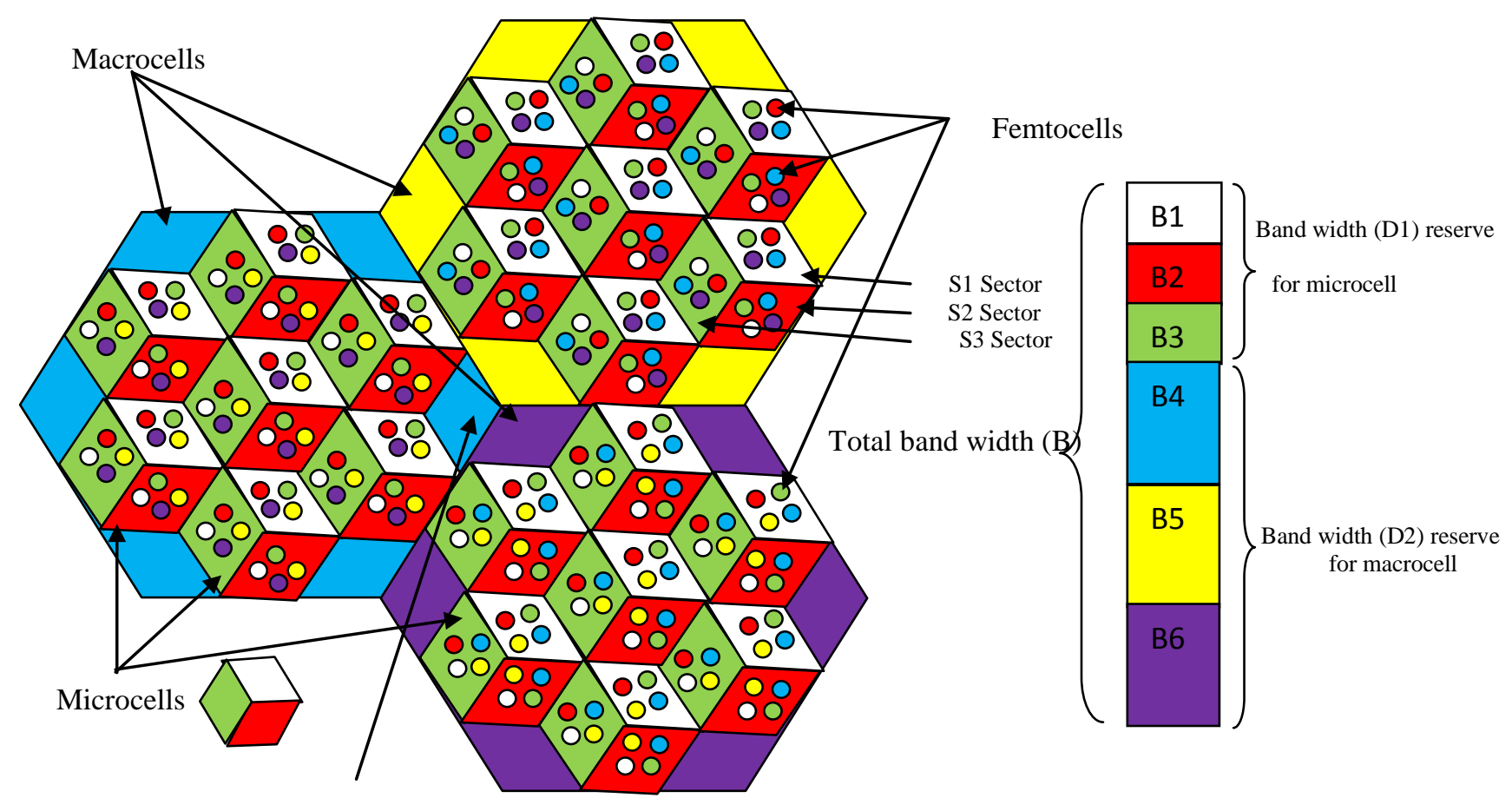

Area only covered by macrocell layer

(a) Three-tier femtocell architecture

(b) Frequency division

Fig. 4 Interference mitigation scheme using FFR in three-tier architecture 
planning to repeat pattern of frequency reuse using 3 , therefore sub band $\mathrm{D} 2$ is also divided into three sub parts $\mathrm{B} 4$ $\mathrm{B} 5$, and B6 satisfying $\mathrm{B} 4+\mathrm{B} 5+\mathrm{B} 6=\mathrm{D} 2$. Now femtocells are deployed within one of the region of macrocell/ microcell as shown in Fig. 4(a). Likewise previous scheme, this scheme femtocell also select those frequency sub band, which are not assigned to microcell sector and macrocell itself as shown in Fig. 4(a). Therefore femtocell applies dissimilar sub band to mitigate the interference from conventional two-tier cellular networks. The sub band is used in the coverage of microcell sector as much as possible. Because femtocell have less transmission power in comparison to conventional cellular communication networks, therefore interference among the different layers of heterogeneous networks is considerably prevented. It has four sub bands to allocate the femtocell in entire microcell region that's why the performance of the system may be better. This same technique is applied to overall networks, therefore, the performance of the entire networks is good. As shown in figure 4(a), area only covered by macrocell layer is created at every point where repeat pattern of frequency reuse using 3 of macrocell is met. At this area, additional microcell may be deployed to create two-tier networks, if required.

Both proposed schemes are suitably and effectively installed in metro/ NCR region to handle the traffic load carefully. The first scheme is suitable for the place where residential/ commercial campus, educational institute are situated towards the highways/link road/ ring road. But, at the place where high speed and slow speed subscribers are located with pedestrian/ staying users, second proposed scheme may be more suitable. Because second scheme have the capability to handle the versatile speed of subscribers simultaneously by assigning the suitable tier with QoS and accommodate both fast speed subscribers and slow speed subscribers simultaneously by reducing handover probability rate of fast speed users.

\section{CONCLUSION AND FUTURE WORK}

The interference management solution would strongly depend on the employed radio access technology (e.g., CDMA or OFDMA) and access mode (i.e., closed, open, or hybrid). In particular, adaptive admission control, power control, interference avoidance and advanced communication strategies such as interference cancellation and beam forming for multiple-antenna transceivers are important techniques to mitigate co-tier and cross-tier interferences. Selection of an interference mitigation scheme depends on the desired tradeoff between complexity and efficiency. This paper is proposed two interference mitigation schemes one for two-tier and other for three-tier femtocell networks since it requires minimal/ no coordination among HeNBs and macrocell/ microcell base station and hence reduces the signaling overhead, and thus the complexity of the system. The proposed schemes are able to mitigate the interference using FFR mechanism. Future work comprises of testing the performance of the proposed interference mitigation schemes through simulation experiments and comparing the results with existing FFR and SFR schemes.

\section{ACKNOWLEDGMENT}

I would like to extend my gratitude and my sincere thanks to my respected, esteemed Director, IET, Mangalayatan University, Aligarh Dr. (Col.) Nagaraj Mantha for his exemplary guidance and encouragement. His trust and support inspired me in the most important moments of making right decisions and I am glad to work under his direction.

\section{REFERENCES}

[1] Vikas Solanki et.at., "Improving the Performance of Handoff Calls using Frequency Sharing”, IJMNCT, Vol. 2(4), August 2012, pp. 71-96.

[2] Vikas Solanki, M. Qasim Rafiq, "Improving the Efficiency of Call Admission Control in Wireless Cellular Communication Networks by Frequency Sharing Techniques", IJCTT, Vol. 9(3), March 2014, pp. 133-146.

[3] V. Chandrasekhar, J. Andrews, and A. Gatherer, "Femtocell networks: a survey," IEEE Communications Magazine, vol. 46, no. 9, Sep. 2008, pp. 59-67.

[4] J. Cullen, "Radio frame presentation," in Femtocell Europe, London, UK, June. 2008.

[5] O.A Akinlabi, B.S. Paul, M. K. Joseph and H.C. Ferreira, "Indoor Communication: Femtocell Behavior in an Indoor Environment", Proceedings of the International MultiConference of Engineers and Computer Scientists, Hong Kong, Vol II, March 18 - 20, 2015.

[6] D. L. Perez, A. Valcarce, G.D. L. Roche, L. Enjie, J. Zhang, "Access methods to WiMAX femtocells: A downlink system level case study", 11th IEEE Singapore International Conference on Communication Systems, 19-21 Nov. 2008, pp.1657-1662.

[7] G. D. I. Roche et al, "Access control mechanisms for femtocells", IEEE Communications Magazine, vol. 48,no. 1, Jan. 2010, pp. 33-39.

[8] Plass, Simon, Doukopoulos, Xenofon G, Legouable, Rodolphe, "Investigations on Link-Level Inter-Cell Interference in OFDMA Systems", Proceedings 13th Annual Symposium on Communications and Vehicular Technology in the Benelux (SCVT 2006), Nov. 2006, pp. 49-52.

[9] Haipeng LEI, Lei ZHANG, Xin ZHANG and Dacheng YANG, "A Novel Multi-Cell OFDMA System Structure using Fractional Frequency Reuse", Proc. IEEE Int. Symposium on Personal, Indoor and Mobile Radio Communications (PIMRC 2007), September 2007.

[10] D. Lopez-Perez, G. de la Roche, A. Valcarce, A. Juttner, and J. Zhang, "Interference Avoidance and Dynamic Frequency Planning for WiMAX Femtocells Networks," Proc. of IEEE Int. Conf. on Commun. Systems (ICS), Nov. 2008.

[11] 3GPP TR R25.820, "3G Home NodeB Study Item," March 2008.

[12] S.S. Prasad and R. Baruah, "Femtocell mass deployment: Indian perspective," 3rd International Conference on Anti-counterfeiting, Security, and Identification in Communication, 20-22 Aug. 2009, pp. 34-37.

[13] H. Chan, C. Hyoung-Kee, and K. In-Hwan, "Building femtocell more secure with improved proxy signature," IEEE Global Telecommunications Conference, Nov. 30Dec. 4 2009, pp. 1-6.

[14]T. Chiba and H. Yokota, "Efficient route optimization methods for femtocell-based all ip networks," IEEE International Conference on Wireless and Mobile Computing, Networking and Communications, 12-14 
Oct. 2009, pp. 221-226.

[15] David L'opez-P'erez, Alvaro Valcarce, Guillaume de la Roche, Jie Zhang, "OFDMA Femtocells: A Roadmap on Interference Avoidance", IEEE Communications Magazine, June 2009, pp. 1 - 8.

[16] 3GPP, "Mobility Procedures for Home NodeB; Overall Description Stage 2," TS 25.367 (release 11), 2011

[17] 3GPP, "Mobility Procedures for Home NodeB; Overall Description Stage 2,” TS 25.367 (release 11), 2011.

[18] S. Ghosh, K. Basu, and S. Das, "An architecture for nextgeneration radio access networks," IEEE Network, vol. 19, no. 5, Sep. 2005, pp. 35-42.

[19] L. Wang, Y. Zhang, and Z. Wei, "Mobility Management Schemes at Radio Network Layer for LTE Femtocells", in Proc. VTC, Barcelona, Spain, Apr. 2009, pp. 1-5.

[20] A. Golaup, M. Mustapha, and L. Patanapongpibul, "Femtocell access control strategy in UMTS and LTE", IEEE Communications Magazine, vol. 47, no. 9, Sep. 2009, pp. 117-123.

[21] 3GPP, "Local IP Access and Selected IP Traffic Offload," TR 23.829 (release 11), 2011.

[22] BeFEMTO - broadband evolved femto networks. [Online]. Available: http://www.ict-befemto.eu/

[23] H.-S. Jo, Y. J. Sang, P. Xia, and J. G. Andrews, "Outage probability for heterogeneous cellular networks with biased cell association," IEEE Globecom, Dec. 2011.

[24] R. Bendlin, V. Chandrasekhar, R. Chen, A. Ekpenyong, and E. Onggosanusi, "From homogeneous to heterogeneous networks: A 3GPP long term evolution rel. 8/9 case study," in CISS, Baltimore, MD, Mar. 2011.

[25] A. Barbieri, A. Damnjanovic, T. Ji, J. Montojo, Y. Wei, D. P. Malladi, O. Song, and G. Horn, "The Downlink Inter-Cell Interference Problem in Rel-10 LTE Femtocell Networks,” IEEE J. Sel. Areas Commun., Apr. 2012.

[26] H. Su, L. Kuang, and J. Lu, "Interference avoidance in OFDMA based femtocell network," IEEE Youth Conference on Information, Computing and Telecommunication, 20-21 Sept. 2009, pp. 126-129.

[27] S.A. Mahmud, G.M. Khan, H. Zafar, K. Ahmad and N Behttani, "A Survey on Femtocells: Benefits Deployment Models and Proposed Solutions", Journal of Applied Research and Technology, vol. 11, Oct. 2013, pp. 733-775.

[28] Talha Zahir, Kamran Arshad, Atsushi Nakata, and Klaus Moessner, "Interference Management in Femtocells", IEEE Communications Surveys \& Tutorials, Vol. 15, No. 1, First Quarter 2013.

[29] Poongup Lee, Taeyoung Lee, Jangkeun Jeong, Jitae Shin, "Interference management in LTE femtocell systems using Fractional Frequency Reuse", Proc. 12th International Conference on Advanced Communication Technology (ICACT), vol.2, 7-10 Feb. 2010, pp. 1047 1051.

[30] N. Saquib, E. Hossain, and D. Kim, "Fractional frequency reuse for interference management in LTEadvanced hetnets", IEEE Wireless Communications, vol. 20, no. 2, Apr. 2013, pp. 113-122.

[31] Tariq, F.; Dooley, L.S.; Poulton, A.S.; Yusheng Ji, "Dynamic fractional frequency reuse based hybrid resource management for femtocell networks", 7th International Conference on Wireless Communications and Mobile Computing (IWCMC), IEEE Conference Publications, 2011, pp. 272-277.

[32] Bouras, C.; Kavourgias, G.; Kokkinos, V.; Papazois, A., "Interference management in LTE femtocell systems using an adaptive frequency reuse scheme", Wireless Telecommunications Symposium (WTS), IEEE Conference Publications, 2012, pp. 1-7.

[33] Sathya, R.V.; Tamma, B.R., "Dynamic spectrum allocation in Femto based LTE network", Fifth International Conference on Communication Systems and Networks (COMSNETS), IEEE Conference Publications, 2013, pp. 1-2.

[34] Sathya, V., Ramamurthy, A. ; Tamma, B.R., "On placement and dynamic power control of femtocells in LTE HetNets", Global Communications Conference (GLOBECOM), 2014 IEEE Conference Publication, 812 Dec. 2014, pp: 4394 - 4399.

[35] M Narsing Yadav, Mounika Palle, Alzayidi Hani, "Performance Analysis of Fractional Frequency Reuse Factor For Interference Suppression In Long Term Evolution", International Journal of Conceptions on Electronics and Communication Engineering, Vol. 3, Issue 1, April’ 2015, pp. 32-35. 